Original Article

\title{
LIPID PROFILE PATTERNS AND ASSOCIATION BETWEEN GLYCATED HAEMOGLOBIN (HbA1C) AND ATHEROGENIC INDEX OF PLASMA (AIP) IN DIABETES PATIENTS AT A TERTIARY CARE HOSPITAL IN MALAYSIA
}

\author{
MUBASHRA BUTT ${ }^{*}$, ADLIAH MHD ALI ${ }^{b}$, MOHD MAKMOR BAKRYc \\ ${ }^{*}$ Faculty of Pharmacy, Universiti Kebangsaan Malaysia, Jalan Raja Muda Abdul Aziz, Kuala Lumpur 50300, Malaysia, bFaculty of Pharmacy, \\ Universiti Kebangsaan Malaysia, Jalan Raja Muda Abdul Aziz, Kuala Lumpur 50300, Malaysia, cFaculty of Pharmacy, Universiti \\ Kebangsaan Malaysia, Jalan Raja Muda Abdul Aziz, Kuala Lumpur 50300, Malaysia \\ Email: mubasyra@gmail.com
}

Received: 29 Aug 2016 Revised and Accepted: 29 Apr 2017

\begin{abstract}
Objective: There is lack of data regarding lipid patterns and the relationship of the atherogenic index of plasma (AIP with glycemic control in Malaysian diabetes patients. This study aimed to assess the lipid patterns and association of AIP with HbA1c in diabetes patients at a tertiary care hospital in Malaysia.
\end{abstract}

Methods: The study was conducted on 72 diabetes patients who were randomly chosen and agreed to participate in the study. AIP values were calculated using log triglycerides/high-density lipoprotein (TG/HDL) ratio. Data were analysed using SPSS 23.

Results: Mean age and body mass index (BMI) of the participants were $56.88 \pm 8.89$ (years) and $28.81 \pm 4.78\left(\mathrm{~kg} / \mathrm{m}^{2}\right)$ respectively. Hypercholesterolemia, hypertriglyceridemia, high low-density lipoprotein (LDL) values were found in $55.5 \%, 41.7 \%, 45.5 \%$ of total participants respectively and less than desirable HDL values were observed in $25 \%$ of total participants. Overall, $37.5 \%$ patients were categorised into high-risk AIP category. BMI categories had a significant association with AIP category $(\mathrm{p}=0.05)$. There was a significant positive correlation of AIP with HbA1c $(r=0.34 ; p=0.004)$. HDL was negatively correlated with AIP $(r=0.27 ; p=0.02)$. Duration of diabetes and ethnicity showed no significant association with AIP risk values.

Conclusion: Diabetes patients in this study exhibited significant lipid abnormalities with increased AIP. AIP was significantly correlated with HbA1c.

Keywords: API, Diabetes Mellitus, HbA1c, Malaysia

(C) 2017 The Authors. Published by Innovare Academic Sciences Pvt Ltd. This is an open access article under the CC BY license (http://creativecommons.org/licenses/by/4.0/) DOI: http://dx.doi.org/10.22159/ijpps.2017v9i6.18639

\section{INTRODUCTION}

Presence of type 2 diabetes is associated with two to four folds increased risk of coronary heart disease (CHD) [1] and leads to decreased quality of life. The gravity of the CHD risk in diabetes was demonstrated in a study which concluded that "diabetes without prior myocardial infarction and prior myocardial infarction without diabetes indicate similar risk for CHD death in men and women" [2]. The National Cholesterol Education Program (NCEP) guidelines classify diabetes mellitus as a coronary artery disease "risk equivalent" therefore diabetes is considered an important risk factor for CHD [3]. Insulin resistance is a core defect in diabetes mellitus that contributes to the development of lipid abnormalities by altering the enzymatic pathways and key enzymes in lipid metabolism, therefore, increasing the risk of CHD [4].

Diabetic atherogenic lipoprotein profile that develops as a result of dyslipidemia is characterised by a high ratio of low-density lipoprotein cholesterol (LDL-C) to high-density lipoprotein cholesterol (HDL-C) and increased level of triglycerides (TG's) [3]. A number of lipoprotein ratios have been used to predict cardiovascular disease (CVD) risks such as LDL cholesterol/apoB ratio, non-HDL cholesterol/HDL cholesterol ratio, apolipoprotein B/apolipoprotein A-I ratio, total cholesterol (TC)/HDL cholesterol and LDL/HDL cholesterol ratios [5]. The atherogenic index of plasma (AIP) is a relatively new indicator of plasma atherogenicity used to predict the risk of CHD and defined as the logarithm of the ratio of plasma triglyceride to HDL-C levels. It is derived from precise measurements of atherogenic lipoprotein profiles namely fractional esterification rate HDL (FERHDL) and LDL particles size and can be used widely as TG and HDL measurements are commonly available [6]. AIP values of $<0.1$ are associated with low cardiovascular risk, values between 0.1 and 0.24 are related to medium cardiovascular risk, and $>0.24$ are related to high cardiovascular risk [7].

Reports on CVD-related mortality in Malaysia showed that CVD contributed to the $32 \%$ of total deaths caused by noncommunicable diseases (WHO 2011) [10]. In 2010, 25.4\% of deaths in Malaysian government hospitals were caused by cardiovascular diseases and it was the leading cause of the premature deaths with about $35 \%$ of CVD-related deaths in occurring in the age group below sixty [11]. Although diabetes is a significant risk factor for CVD in Malaysia, there is a paucity of data on lipid profile patterns and API studies amongst diabetes patients in local healthcare setup. Studies that investigate the lipid patterns, the association between glycemic control, AIP and patients' characteristics can facilitate the understanding of CHD risk in indigenous diabetes patients. The aim of this study was to evaluate the lipid patterns and association between HbA1c and AIP values in type 2 diabetes patients at a tertiary care hospital in Malaysia.

\section{MATERIALS AND METHODS}

The present study analysed the data of diabetes patients from a randomised controlled study conducted at a tertiary care hospital in Malaysia [12]. The study was approved by the University Kebangsaan Malaysia's research ethics committee (UKMREC) (Ref No. UKM 1.5.3.5/244/NF-018-2012). Patients recruited were indigenous Malaysians who agreed to be part of the study and signed the consented forms. Dyslipidemia was defined using National Cholesterol Education Program - Adult Treatment Panel III (NCEP - ATP III). Body mass index (BMI) was derived from weight and height of the participants and BMI categories were based on WHO classification. Healthy weight category had BMI values 18.50 - 
$24.99 \mathrm{~kg} / \mathrm{m}^{2}$. Overweight category range was 25 - 29.99 $\mathrm{kg} / \mathrm{m}^{2}$ and obesity was categorised as having $\geq 30 \mathrm{~kg} / \mathrm{m}^{2} \mathrm{BMI}$ values. Data were entered and analysed using SPSS version 23 For descriptive analysis, sociodemographic and clinical data of participants were presented as a percentage, mean and standard deviations. Pearson's correlation test was performed to assess the various correlations and independent sample T-Test and ANOVA were applied to compare means between various groups. Pearson's chi-square and Fisher exact test were performed to find out the correlation between categorical variables. All necessary statistical assumptions were checked and compiled before running all SPSS tests. Missing data were imputed using SPSS imputation command.

HbA1c was used as an outcome to study the glycemic control of the participants. Currently, it is the best marker to reflect the glycemic control of the diabetes patients during the past 6 to 8 w. The value of HbA1c correlates well with the circulating blood glucose levels [13].

AIP was calculated by using base 10 logarithms of the ratio of TG to HDL, formula $=\log (\mathrm{TG} / \mathrm{HDL})$. Patients were classified into low/medium risk and high-risk group based on their AIP values. Low/medium risk group included participants with AIP values less than 0.24 and the high-risk group had participants with AIP values more than 0.24 [7].

\section{RESULTS}

Our study included a total of 72 diabetes patients, of which 41 percent were male and 58.3 percent were female. Mean age of the participants was $56.88 \pm 8.89 \mathrm{y}$. The mean BMI for the participants was $28.81 \pm 4.78 \mathrm{~kg} / \mathrm{m}^{2} ; 27$ patients in our study were classified as obese according to WHO classification (BMI $>30 \mathrm{~kg} / \mathrm{m}^{2}$ ). Among the 72 participants, 41 were Malay, 17 were Indian and 14 were Chinese. No significant differences in lipid profiles of the patients were found between male and female participants. Results of HbA1c assay showed only 9 patients had a controlled value of less than 7 percent. Table 1 and 2 entail the detailed results of demographic and biochemical profiles of the patients. Among all the participants, hypercholesterolemia was found in $55.5 \%$, high LDL values in $45.5 \%$, less than desirable HDL values in $25 \%$, and hypertriglyceridemia in $41.7 \%$ of the total participants. Table 3 shows the results of correlation analysis of glycaemic control and lipid profiles. There was a significant and positive correlation between AIP values, triglycerides and HbA1c values. HDL profile had a negative correlation with $\mathrm{HbA1c}(\mathrm{p}$-value $=0.054)$. Analysis of lipid parameters using ANOVA revealed there were no significant differences in lipid parameters amongst ethnicities. Sub-analysis of BMI groups indicated non-significant differences between glycaemic control of the patients in three categories (healthy weight $=9.23 \pm 1.22$, overweight $=9.12 \pm 1.68$, obese $=$ $8.63 \pm 1.49 ; \mathrm{p}=0.32$ ).

Table 1: Demographic characteristics of the participants and their classification according to AIP values

\begin{tabular}{|c|c|c|c|c|c|}
\hline Characteristics & All (n= 72) & Low $/$ med risk $(n=45)$ & High risk $(n=27)$ & †Total & P value \\
\hline & & $45(62.5 \%)$ & $27(37.5 \%)$ & & \\
\hline Age (y) & $56.88 \pm 8.89$ & $56.51 \pm 8.46$ & $57.70 \pm 9.77$ & & 0.58 \\
\hline \multicolumn{6}{|l|}{ Gender } \\
\hline Male & $30(41.7 \%)$ & $16(53.3 \%)$ & $14(46.7 \%)$ & $100 \%$ & 0.22 \\
\hline Female & $42(58.3 \%)$ & $29(69 \%)$ & $13(31 \%)$ & $100 \%$ & \\
\hline BMI $\left(\mathrm{kg} / \mathrm{m}^{2}\right)$ & $28.81 \pm 4.78$ & $28.66 \pm 4.26$ & $28.90 \pm 5.64$ & & 0.83 \\
\hline Healthy & $22(30.6 \%)$ & $11(50 \%)$ & $11(50 \%)$ & $100 \%$ & $0.05^{*}$ \\
\hline Over weight & $23(31.9 \%)$ & $19(82.6 \%)$ & $4(17.4 \%)$ & $100 \%$ & $\left(X^{2}=5.99\right)$ \\
\hline Obese & $27(37.5 \%)$ & $15(55.6 \%)$ & $12(44.4 \%)$ & $100 \%$ & \\
\hline \multicolumn{6}{|l|}{ Ethnicity } \\
\hline Malay & $41(56.9 \%)$ & $22(53.7 \%)$ & $19(46.3 \%)$ & $100 \%$ & 0.12 \\
\hline Indian & $17(23.6 \%)$ & $14(82.4 \%)$ & $3(17.6 \%)$ & $100 \%$ & \\
\hline Chinese & $14(19.4 \%)$ & $9(64.3 \%)$ & $5(35.7 \%)$ & $100 \%$ & \\
\hline \multicolumn{6}{|c|}{ Duration of diabetes (y) } \\
\hline$<5$ & $18(25.0 \%)$ & $12(66.7 \%)$ & $6(33.3 \%)$ & $100 \%$ & 0.64 \\
\hline 5 to 10 & $27(37.5 \%)$ & $18(66.7 \%)$ & $9(33.3 \%)$ & $100 \%$ & \\
\hline More than 10 & $27(37.5 \%)$ & $15(55.6 \%)$ & $12(44.4 \%)$ & $100 \%$ & \\
\hline
\end{tabular}

*Statistically significant at $\mathrm{p} \leq 0.05$; values are expressed as mean \pm SD for age and BMI and their $\mathrm{p}$ values are derived from T-test; other $\mathrm{p}$ values are from Pearson's chi square test; med = medium, †Total column contains the percentages of low/medium risk and high risk columns added together, Number of experiments $(n=1)$

Table 2: Lipid profiles parameters categorized by AIP risk values

\begin{tabular}{|c|c|c|c|c|c|}
\hline Characteristics & All (n= 72) & Low $/$ med risk $(n=45)$ & High risk $(n=27)$ & †Total & P value \\
\hline HbA1c \% & $8.92 \pm 1.53$ & $8.84 \pm 1.40$ & $9.18 \pm 1.63$ & & 0.35 \\
\hline$<7$ & $9(12.5 \%)$ & $6(66.7 \%)$ & $3(33.3 \%)$ & $100 \%$ & 0.78 \\
\hline$>7$ & $63(87.5 \%)$ & $39(61.9 \%)$ & $24(39.1 \%)$ & $100 \%$ & \\
\hline $\mathrm{TC} \mathrm{mmol} / \mathrm{l}$ & $5.28 \pm 1.45$ & $5.15 \pm 1.42$ & $5.53 \pm 1.51$ & & 0.29 \\
\hline$<5.2$ & $40(55.5 \%)$ & $28(70 \%)$ & $12(30 \%)$ & $100 \%$ & 0.14 \\
\hline$>5.2$ & $32(45.5 \%)$ & $17(53.1 \%)$ & $15(46.9 \%)$ & $100 \%$ & \\
\hline LDL mmol/l & $3.11 \pm 1.22$ & $3.03 \pm 1.28$ & $3.27 \pm 1.13$ & & 0.42 \\
\hline$<2.6$ & $32(45.5 \%)$ & $23(71.9 \%)$ & $9(28.1 \%)$ & $100 \%$ & 0.14 \\
\hline$>2.6$ & $40(55.5 \%)$ & $22(55 \%)$ & $18(45 \%)$ & $100 \%$ & \\
\hline HDL mmol/l & $1.37 \pm 0.40$ & $1.52 \pm 0.35$ & $1.09 \pm 0.32$ & & $<0.0001^{*}$ \\
\hline$>1$ & $54(75 \%)$ & $39(72.2 \%)$ & $15(27.8 \%)$ & $100 \%$ & $<0.0001^{*}$ \\
\hline$<1$ & $18(25 \%)$ & $6(33.3 \%)$ & $12(66.7 \%)$ & $100 \%$ & \\
\hline TGs mmol/l & $1.69 \pm 0.99$ & $1.27 \pm 0.46$ & $2.50 \pm 1.08$ & & $<0.0001^{*}$ \\
\hline$<1.7$ & $42(58.3 \%)$ & 37 (88.1\%) & $5(11.9 \%)$ & $100 \%$ & $<0.0001^{*}$ \\
\hline$>1.7$ & $30(41.7 \%)$ & $8(26.7 \%)$ & $22(73.3 \%)$ & $100 \%$ & \\
\hline
\end{tabular}

*Statistically significant; values are reported as mean \pm SD and percentages; $\mathrm{TC}=$ total cholesterol; med = medium, ${ }^{+}$Total column contains the percentages of low/medium risk and high risk columns added together, Number of experiments $(n=1)$. 
Table 3: Lipid profiles parameters characterized by the gender

\begin{tabular}{|c|c|c|c|c|}
\hline \multirow[t]{2}{*}{ Parameter } & \multirow[t]{2}{*}{ Targets } & \multicolumn{2}{|c|}{ Means values } & \multirow[t]{2}{*}{ P value } \\
\hline & & Males & Females & \\
\hline Age (years) & & $58.66 \pm 7.00$ & $55.74 \pm 9.98$ & 0.17 \\
\hline $\mathrm{TC} \mathrm{mmol} / \mathrm{l}$ & $<5.2$ & $5.05 \pm 1.29$ & $5.47 \pm 1.55$ & 0.23 \\
\hline LDL mmol/l & $<2.6$ & $2.93 \pm 0.96$ & $3.25 \pm 1.38$ & 0.28 \\
\hline HDL mmol/l & & $1.28 \pm 0.34$ & $1.42 \pm 0.43$ & 0.16 \\
\hline Males & $>1$ & & & \\
\hline Females & $>1.2$ & & & \\
\hline TG mmol/l & $<1.7$ & $1.74 \pm 1.06$ & $1.73 \pm 0.89$ & 0.97 \\
\hline $\mathrm{HbA} 1 \mathrm{c} \%$ & $<7 \%$ & $9.07 \pm 1.68$ & $8.89 \pm 1.35$ & 0.63 \\
\hline BMI $\left(\mathrm{kg} / \mathrm{m}^{2}\right)$ & & $28.63 \pm 5.62$ & $28.83 \pm 4.18$ & 0.86 \\
\hline
\end{tabular}

Number of experiments $(n=1)$; values are expressed as mean \pm SD

Table 4: Correlation studies between the blood glucose and serum lipid profile variables of the participants

\begin{tabular}{lll}
\hline Parameter & HbA1c r (P value) & BMI r (P value) \\
\hline API & $0.34^{*}(0.004)$ & $0.11(0.36)$ \\
TC & $0.10(0.39)$ & $-0.12(0.31)$ \\
LDL & $0.98(0.41)$ & $-0.14(0.25)$ \\
HDL & $-0.23(0.054)$ & $0.07(0.52)$ \\
TG & $0.27^{*}(0.02)$ & $-0.13(0.29)$ \\
BMI & $-0.25^{*}(0.04)$ & \\
\hline
\end{tabular}

*Statistically significant at $\mathrm{p} \leq 0.05 ; \mathrm{r}=$ Pearson's correlation coefficient

\section{DISCUSSION}

In our study $58.3 \%$ of total participants were females and $41.7 \%$ were males. Women with diabetes have a higher risk for cardiovascular complications; the risk of fatal CHD is $50 \%$ greater in female diabetes patients than their male counterparts [14]. However, in our study subanalysis revealed a higher percentage of total females (69\%) in low risk group and a higher proportion of total males $(53.3 \%)$ in highrisk group. In view of the observation that values of AIP correlated positively with the HbA1c value in our study (table 4), a greater percentage of males in the high-risk group can be attributed to a higher mean HbA1c value in males compared to the females (table 3).

The analysis in table 2 shows the values of total cholesterol, HDL, and LDL were higher in females compared to their male counterparts; however, the difference did not reach statistical significance. This finding is similar to the previous studies reporting higher mean values of the aforementioned lipid variables in females $[15,16]$. The lipoprotein metabolism variations during the course of men and women's lives present as the differences in CHD risks at various stages of life. The lipoprotein differences between both the sexes are hypothesised to be partially responsible for the lower risk of cardiovascular events observed in premenopausal women as compared with similarly aged men [17]. However, postmenopausal women are reported to have different and more atherogenicity prone lipid profiles than premenopausal women [18]. In our study the mean age of women fell in the postmenopausal range (more than 40 y) therefore raised lipid parameters may be explained by postmenopausal changes together with a better glycemic control in females than the male counterparts in this study (although the difference was statistically non-significant).

Duration of diabetes and ethnicity showed no significant association with AIP risk values. Previous studies reveal mixed results; some showed no or little role of diabetes duration in determining the risk of dyslipidemia and CHD [19, 20], others demonstrated a positive correlation of duration with CHD risk [21, 22]. Previously, Davis et al. and Zhang et al. found important ethnic differences in lipid profiles of diabetes patients, their results showed distinct patterns of lipid profiles amongst various ethnicities [23, 24]. In the past, Malaysian studies showed mixed results with some studies showing no significant ethnic preference for dyslipidemia in diabetes patients $[25,26]$ and others reporting a significant preference [27, 28]. Regarding AIP, studies that evaluate the AIP amongst Malaysian ethnicities are unavailable for comparison due to paucity of data. We recommend more such studies to ascertain the ethnic differences in CHD risks using AIP values.

Of the total 72 patients, 45 (62.5\%) were categorized as medium/low risk and 27 (47.5\%) as high risk patients. Correlation analysis showed a significant positive relationship between HbA1c and AIP values. These results suggest an association between the appearance of increased CHD risk and hyperglycaemia. It may be hypothesised that worsening of glycemic control reflected by HbA1c may also independently reflect the increased CHD risk. It supports the previous data regarding the use of HbA1c as a potential biomarker of CHD risk [16]. The results are in agreement with previous studies that found a similar association between glycemic control and cardiovascular risk predicted by lipid profiles $[16,29]$. High-risk group had a higher HbA1c value $(9.18)$ than low risk group (8.84). Amongst the patients with $<7 \% \mathrm{HbA} 1 \mathrm{c}, 66.7 \%$ fell in the low/medium risk category compared to $61.9 \%$ of patients with $>7$ $\% \mathrm{HbA} 1 \mathrm{c}$ in the same category. However, both these differences were statistically non-significant. One reason for non-significance could be the small proportion of patients with $<7 \% \mathrm{HbA1c}$, only 9 patients in total had maintained the target of $<7 \%$ HbA1c. These observations reaffirm that a better glycemic control in diabetes leads to a better lipid profile, therefore, decreasing the CHD risk.

In our study, overweight patients significantly took dominance in low risk category and there was no significance correlation between AIP, TC, HDL, LDL, TG, and BMI. On the contrary, other studies have shown a positive correlation of BMI with AIP and/or lipid profiles $[30,31]$. As part of the routine care, the patients in the present study setting were referred to a dietician if they experienced difficulties with their weight control. The more favourable AIP risk scores of overweight patients in our study can be partially explained by the advised lifestyle changes and diet modifications delivered by their dietician in view of their increasing weights. Further sub analysis revealed that there was a negative correlation between glycaemic control and BMI in the present study, patients in healthy weight category had the highest $\mathrm{HbA1c}$ (healthy weight $=9.23 \pm 1.22$, overweight $=9.12 \pm 1.68$, obese $=8.63 \pm 1.49 ; \mathrm{p}=0.32$ ). Although the difference was non-significant, it supports our notion that patients in overweight and obese categories received a relatively intense advice to control their disease. It is not unlikely that overweight patients would be more concerned about their health risks than healthy weighing patients and would seek lifestyle advice more actively. However, obesity is an established risk of coronary heart disease and patients should be advised to maintain a healthy BMI regardless of their risk scores calculated at one point of time only. 
Our results reflect that most common lipid abnormality in the participants was LDL elevation (55.5\%) followed by TC (45.5\%) and TG elevation (41.7\%). Reduced HDL was observed in $25 \%$ patients. These results are supported by the previous studies that demonstrated lipid abnormalities in diabetes patients $[27,19,29$, 16]. As shown in table 2, TC and LDL values were higher in the highrisk category than low/medium risk category although the difference was not statistically significant $(\mathrm{p}=0.42)$. There is a possibility that substantial differences might not have been detected due to the inadequacy of sample size. This observation shows more studies with larger sample size should be conducted at multiple centres to evaluate the results with more statistical power. Among these parameters, only TG was significantly correlated with HbA1c in our study. HDL showed a negative correlation with $\mathrm{HbA1c}$ but $\mathrm{p}$ value failed to reach statistical significance $(p=0.054)$. This suggests that association of CHD risk and glycemic control can be reflected by the $\log$ (TG/HDL) ratio apart from individual lipid parameters. Recent studies have supported the usefulness of AIP value to predict CVD risk and shown it to be a reliable predictor of CHD [30,31]. The presence of lipid abnormalities renders the diabetes patients more prone to cardiovascular risk and cerebrovascular diseases; therefore, it is essential that diabetes patients and caregiver work together to chalk out appropriate interventions for the management of dyslipidemia. In addition, previous studies have provided evidence that the cost of diabetes with complications produces almost double burden compared to the presence of diabetes alone [32]. Better management of dyslipidemia can prove helpful in reducing the healthcare burden.

\section{Limitations of the study}

This study had certain limitations; there was no investigation of the influence of various confounding factors on the correlation of $\mathrm{HbA} 1 \mathrm{c}$ with AIP. This study had the relatively smaller sample size and included the majority of poorly controlled diabetes patients. Studies with improved experimental design, a control group and larger sample size must be conducted in future to further investigate the associations observed in the present study. However, it can serve as the pilot study for such investigations.

\section{CONCLUSION}

Lipid abnormalities are frequently found in diabetes patients and are associated with increased risk of CHD. The most common lipid abnormality in the participants was LDL elevation (55.5\%) followed by TC (45.5\%) and TG elevation (41.7\%). AIP is a relatively new CHD risk predictor; our findings indicated that according to AIP categorization $37.5 \%$ participants were at a high risk of CHD in the present study. AIP was significantly correlated with HbA1c values in diabetes patients. Overweight patients took dominance in the low risk category. No association was observed between duration, age, ethnicity and AIP values.

\section{CONTRIBUTION OF AUTHORS}

We declare that all three authors have made substantial contributions to conception and design, acquisition, analysis, and interpretation of data. All authors have participated in drafting and revising the article and have given their approval for submission and intellectual content.

\section{Compliance with ethical standards}

All procedures performed in the present study involving human participants were in accordance with the ethical standards of the UKMREC (Ref. No. UKM 1.5.3.5/244/NF-018-2012). Informed consent was obtained from all individual participants included in the study. The authors have no conflict of interest to disclose.

\section{CONFLICT OF INTERESTS}

\section{Declared none}

\section{REFERENCES}

1. Haffner SM, Association AD. Management of dyslipidemia in adults with diabetes. Diabetes Care 2003;26:S83.
2. Juutilainen A, Lehto S, Rönnemaa T, Pyörälä K, Laakso M. Type 2 diabetes as a "Coronary Heart Disease Equivalent" an $18 \mathrm{y}$ prospective population-based study in finnish subjects. Diabetes Care 2005;28:2901-7.

3. Expert Panel on Detection E. Executive summary of the Third Report of the National Cholesterol Education Program (NCEP) expert panel on detection, evaluation, and treatment of high blood cholesterol in adults (Adult Treatment Panel III). JAMA 2001;285:2486.

4. Taskinen MR. Diabetic dyslipidemia. Atheroscler Suppl 2002;3:47-51.

5. Masana L, Mangas A, Hernndez-Mijares A, Gonzlez-Santos P. Lipoprotein ratios: physiological significance and clinical usefulness in cardiovascular prevention. Vasc Health Risk Manage 2009;5:1033-42.

6. Dobiášová M, Frohlich J. The plasma parameter log (TG/HDL-C) as an atherogenic index: correlation with lipoprotein particle size and esterification rate inapoB-lipoprotein-depleted plasma (FER HDL). Clin Biochem 2001;34:583-8.

7. Dobiasova M. AIP-- an atherogenic index of plasma as a significant predictor of cardiovascular risk: from research to practice. Vnitrni Lekarstvi 2006;52:64-71.

8. Selvin E, Marinopoulos S, Berkenblit G, Rami T, Brancati FL, Powe NR, et al. Meta-analysis: glycosylated hemoglobin and cardiovascular disease in diabetes mellitus. Ann Intern Med 2004;141:421-31.

9. Selvin E, Steffes MW, Zhu H, Matsushita K, Wagenknecht L, Pankow J, et al. Glycated hemoglobin, diabetes, and cardiovascular risk in nondiabetic adults. $\mathrm{N}$ Engl J Med 2010;362:800-11.

10. World Health Organization WHO; 2011. Available from: http://www.who.int/nmh/countries/mys_en.pdf. [Last accessed on 07 Jun 2016]

11. Malaysia MoH. Ministry of Health Malaysia. Annual Report Putrajaya; 2010.

12. Butt M, Ali AM, Bakry MM, Mustafa N. Impact of a pharmacist led diabetes mellitus intervention on HbA1c, medication adherence and quality of life: a randomised controlled study. Saudi Pharm J 2016;24:40-8.

13. World Health Organization. Use of glycated haemoglobin (HbA1c) in the diagnosis of diabetes mellitus: abbreviated report of a WHO consultation. InUse of glycated haemoglobin (HbA1c) in the diagnosis of diabetes mellitus: abbreviated report of a WHO consultation; 2011.

14. Huxley R, Barzi F, Woodward M. Excess risk of fatal coronary heart disease associated with diabetes in men and women: a meta-analysis of 37 prospective cohort studies. Br Med J 2006;332:73-8.

15. Wexler DJ, Grant RW, Meigs JB, Nathan DM, Cagliero E. Sex disparities in treatment of cardiac risk factors in patients with type 2 diabetes. Diabetes Care 2005;28:514-20.

16. Vinod Mahato R, Gyawali P, Raut PP, Regmi P, Singh KP, Raj Pandeya D, et al. Association between glycaemic control and serum lipid profile in type 2 diabetic patients: glycated haemoglobin as a dual biomarker. Biomed Res 2011;22:375-80.

17. Bittner V. Lipoprotein abnormalities related to women's health. Am J Cardiol 2002;90:77-84.

18. Warren MP, Halpert S. Hormone replacement therapy: controversies, pros and cons. Best Pract Res Clin Endocrinol Metab 2004;18:317-32.

19. Dixit AK, Dey R, Suresh A, Chaudhuri S, Panda AK, Mitra A, et al. The prevalence of dyslipidemia in patients with diabetes mellitus of ayurveda Hospital. J Diabetes Metab Disord 2014;13:58.

20. Okpa HO, Enang OE, Effa EE, Essien OE, Mbu PN. Comparative analysis of the atherogenic index of plasma and its relationship with cardiovascular risk among patients with diabetes mellitus and concurrent diabetes mellitus with hypertension attending endocrinology clinic in a tertiary hospital South-South Nigeria. IOSR J Dental Med Sci 2015;14:102-7.

21. Otamere H, Aloamaka C, Okokhere P, Adisa W. Lipid profile in diabetes mellitus; what impact has age and duration. $\mathrm{Br} \mathrm{J}$ Pharmacol Toxicol 2011;2:135-7. 
22. Patel MB, Sachora WM, Pandya AR, Kothari AD, Patel JK. Can HbA1c Act as a surrogate marker for cardiovascular risk? IOSR J Dental Med Sci 2013;3:39-43.

23. Davis TM, Cull CA, Holman RR. The relationship between ethnicity and glycemic control, lipid profiles, and blood pressure during the first $9 \mathrm{Y}$ of type 2 diabetes UK prospective diabetes study (UKPDS 55). Diabetes Care 2001;24:1167-74.

24. Zhang L, Qiao Q, Tuomilehto J, Janus ED, Lam TH, Ramachandran A, et al. Distinct ethnic differences in lipid profiles across glucose categories. J Clin Endocrinol Metab 2010;95:1793-801.

25. Blebil AQ, Hassan Y, Dujaili JA. Differences in demographics, lipid profile and other clinical characteristics among type 2 diabetic patients in the state of penang, Malaysia according to gender and races. Asian J Pharm Clin Res 2011;4 Suppl 2:130-3.

26. Samsudin IN, Md Saleh R, Chellappah Thambiah S, Hamzah MA, Syafira A, Khalik W, et al. Prevalence of dyslipidaemia in type 2 diabetes mellitus patients and its association to diabetic retinopathy in a Malaysian tertiary hospital. Malaysian J Med Health Sci 2014;10:47-51.

27. Chew BH, Ismail M, Lee PY, Taher SW, Haniff J, Mustapha FI, et al. Determinants of uncontrolled dyslipidaemia among adult type 2 diabetes in Malaysia: the Malaysian diabetes registry. Diabetes Res Clin Pract 2012;96:339-47.
28. Ismail IS, Nazaimoon W, Mohamad $\mathrm{W}$, Letchuman $\mathrm{R}$ Singaraveloo M, Hew F, et al. Ethnicity and glycemic control are major determinants of diabetic dyslipidaemia in Malaysia. Diabetic Med 2001;18:501-8.

29. Ozder A. Lipid profile abnormalities seen in T2DM patients in primary healthcare in Turkey: a cross-sectional study. Lipids Health Dis 2014;13:183.

30. Nansseu JRN, Moor VJA, Nouaga MED, Zing-Awona B, Tchanana $G$, Ketcha A. Atherogenic index of plasma and risk of cardiovascular disease among Cameroonian postmenopausal women. Lipids Health Dis 2016;15:41.

31. Niroumand S, Khajedaluee M, Khadem-Rezaiyan M, Abrishami M, Juya M, Khodaee G, et al. Atherogenic index of plasma (AIP): a marker of cardiovascular disease. Med J Islam Repub Iran 2015;29:240.

32. Konuru V, Sangam K, Mohammed A, Kanneganti S. Evaluation of pharmacoeconomic direct cost in diabetes patients. Asian J Pharm Clin Res 2017;10:38-40.

\section{How to cite this article}

- $\quad$ Mubashra Butt, Adliah Mhd Ali, Mohd Makmor Bakry. Lipid profile patterns and association between glycated haemoglobin (HbA1C) and atherogenic index of plasma (AIP) in diabetes patients at a tertiary care hospital in Malaysia. Int J Pharm Pharm Sci 2017;9(6):150-154. 